\title{
Legionella Pneumonia: When to Suspect, Diagnostic Considerations, and Treatment Strategies for Hospital-Based Clinicians
}

\author{
Robert Eison
}

Published online: 9 October 2014

(C) Springer Science+Business Media New York 2014

\begin{abstract}
Legionella species are fastidious gram-negative bacteria, ubiquitous in nature and associated with two distinct clinical syndromes: Legionnaire's disease and Pontiac fever. Legionnaires' disease is a severe multisystem disease comprised of fevers, rigors, headache, myalgias, dyspnea, delirium, and diarrhea, whereas Pontiac fever is a self-limited influenza-like illness. Either syndrome can occur during outbreaks, but typically only Legionnaires' disease and pneumonia come to the attention of the emergency room physician or hospitalist. Here we describe an illustrative case of a 70-year-old female with COPD who presented to our hospital with severe Legionnaires' disease. This article reviews the epidemiology and clinical manifestations of Legionnaires' disease and updates the latest information on diagnostic approaches and treatment strategies for hospital-based clinicians and emergency department physicians alike.
\end{abstract}

Keywords Legionella - Legionellosis - Legionnaire's disease $\cdot$ Pontiac fever $\cdot$ Atypical pneumonia $\cdot$ Communityacquired pneumonia

\section{Case}

A 70-year-old Caucasian female with a history of COPD presented with 5 days of progressive shortness of breath, fevers, and cough. She was found to have significant hypoxemia, fever to $38.9^{\circ} \mathrm{C}$, leukocytosis of 22,000

R. Eison $(\square)$

Colorado Infectious Disease Associates, 950 East Harvard Ave

Suite 140, Denver, CO 80210, USA

e-mail: rceisonmd@gmail.com [cells $\times 10^{9} / \mathrm{L}$ ] and was treated with ceftriaxone and azithromycin. Unfortunately, she deteriorated with worsening hypoxemia, hypotension, and tachycardia. She required intubation as well as norepinephrine for blood pressure support. Azithromycin was continued but ceftriaxone was discontinued in favor of vancomycin and piperacillin/tazobactam. Imaging revealed a dense right lower lobe consolidation (Figs. 1-4). Bronchoalveolar lavage cultures were unrevealing but Legionella urinary antigen returned positive. Levofloxacin was started at $750 \mathrm{mg}$ once daily and the other antibiotics were discontinued. She improved steadily thereafter and made a full recovery, returning home one week later and completing 14 days of levofloxacin. She resides in the mountains on a large parcel of land and reported that she has diverted water from a nearby creek and maintains a water heater for her shower using this creek water. She also reported spending time recently in a friend's hot tub. The State health department did not find evidence of Legionella in the patient's water heater or the nearby creek. However, the hot tub in question was not included in the investigation.

\section{Legionella}

Legionella species are fastidious gram-negative bacteria, ubiquitous in nature as intracellular parasites within freshwater protozoa. Although isolated originally in 1943 where it was discovered residing within free-living amoebae in the soils of Poland [1], it only achieved notoriety as a pathogen in 1976 during the pneumonia epidemic at the American Legion convention in Philadelphia, PA when this gram-negative organism was isolated from the lung tissue of an autopsied victim [2]. CDC investigators later traced this organism back to another outbreak in Pontiac, Michigan in 1968 demonstrating increased Legionella titers in 


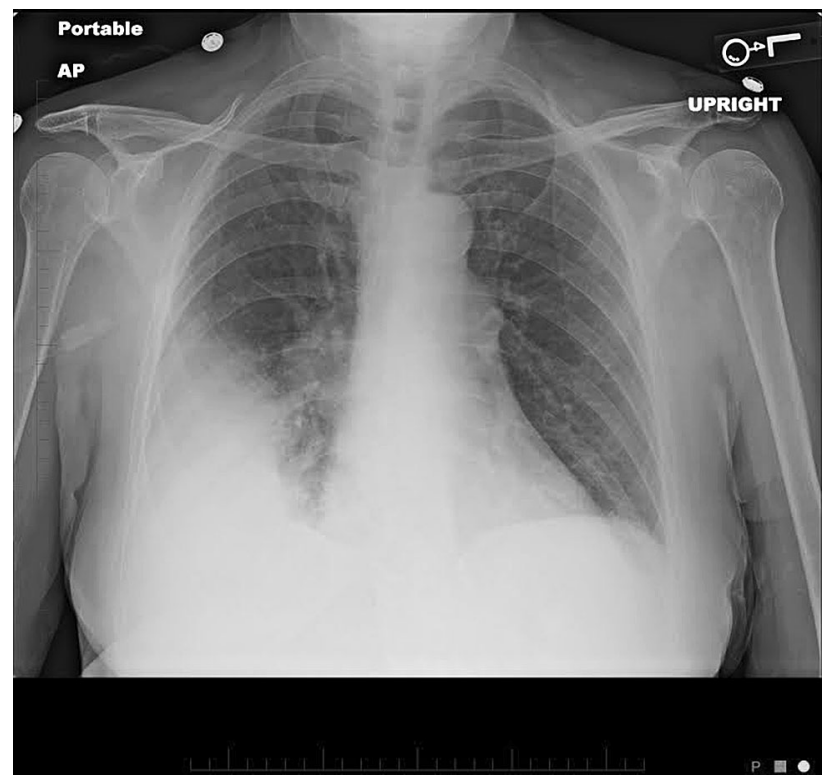

Fig. 1 Admission chest X-ray demonstrating a right lower lob consolidation

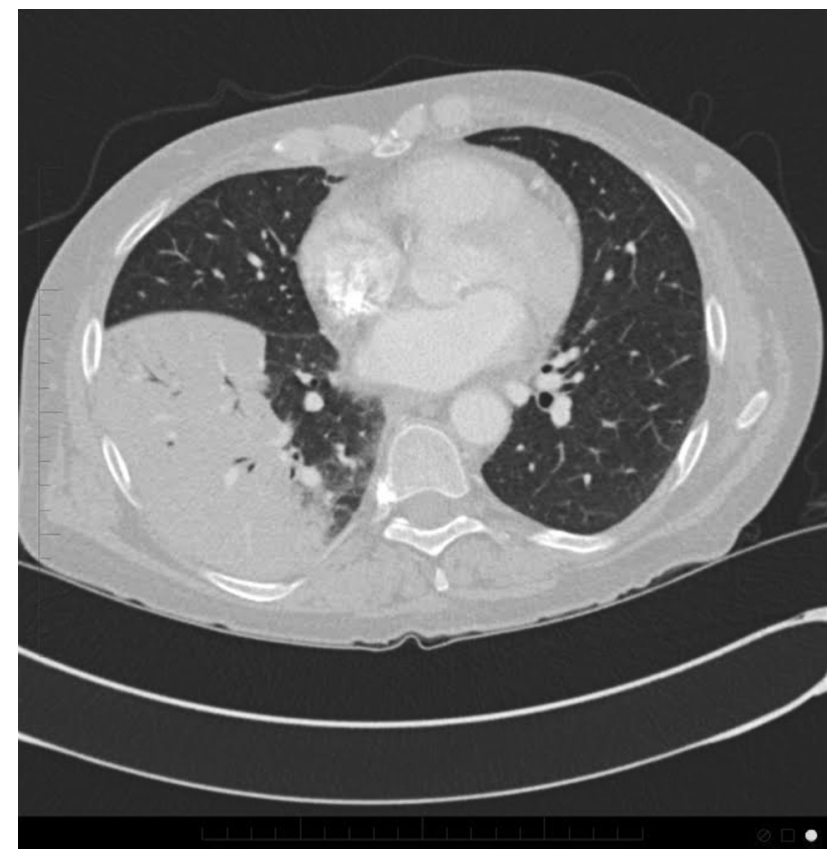

Fig. 2 Chest $\mathrm{CT}$ axial view demonstrating the dense right lower lobe consolidation

survivors' stored sera. Since then it has become increasingly identified as the scourge in innumerable pneumonia outbreaks as well as the causative agent between 2 and $15 \%$ of hospitalized cases of community-acquired pneumonia [3]. Global incidence has steadily risen both in epidemic outbreaks and sporadic cases. In the US alone, the annual incidence of reported cases climbed $217 \%$ between 2000 and 2009 with the actual incidence felt to be much higher [4].

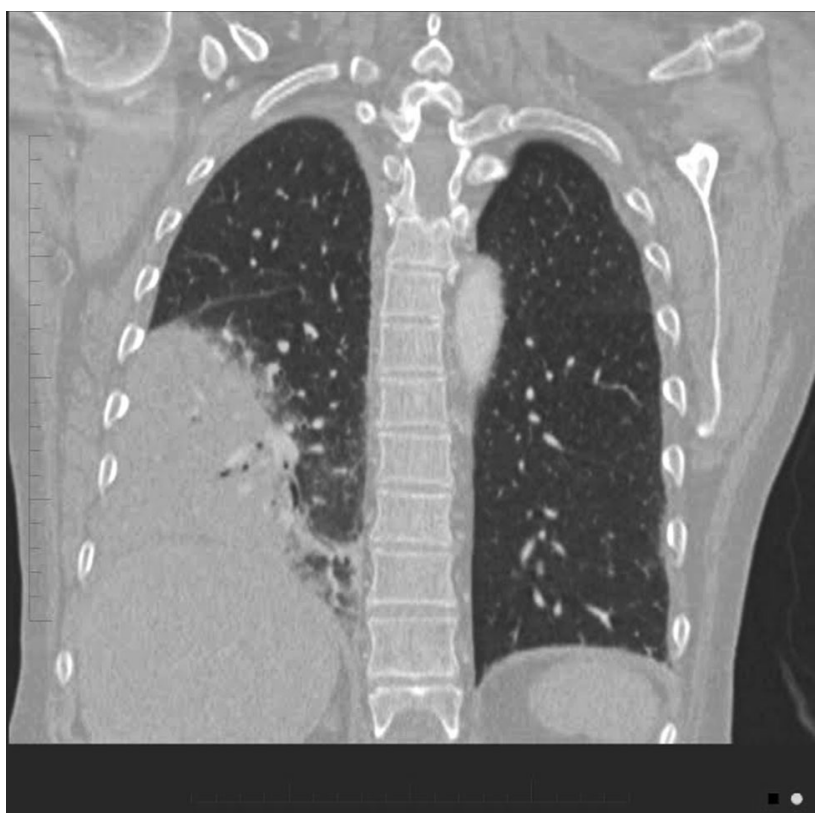

Fig. 3 Chest CT coronal view

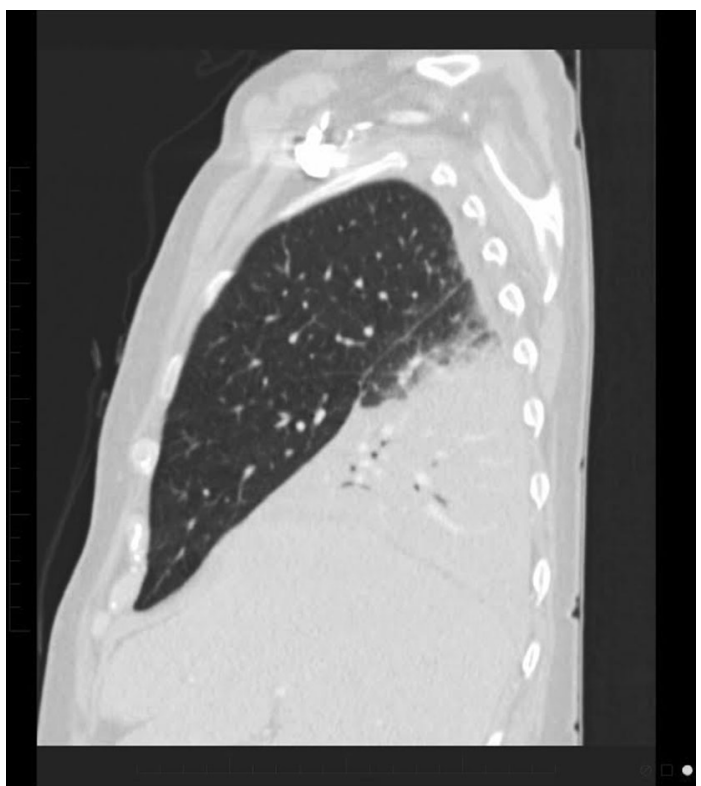

Fig. 4 Chest CT sagittal view demonstrating near complete opacification of the right lower lobe

Classically, Legionella is associated with two distinct clinical syndromes whose monikers result from the two aforementioned epidemics: Legionnaire's disease and Pontiac fever. Legionnaires' disease is a severe multisystem disease comprised of fevers, rigors, headache, myalgias, dyspnea, delirium, and diarrhea, whereas Pontiac fever is a self-limited influenza-like illness. Either syndrome can occur during outbreaks, but typically only Legionnaires' disease and pneumonia come to the attention 
of the Emergency room physician or hospitalist. Clinicians commonly attribute "atypical pneumonia" to one of the triumvirate of intracellular respiratory bacterial pathogens Legionella, Mycoplasma pneumoniae, and Chlamydophila pneumonia, though differentiating these entities from each other or even from "typical pneumonias" such as Pneumococcal pneumonia is inherently fraught with challenges. However, a comprehensive and incisive approach utilizing a "clinical syndromic diagnosis" espoused by some authors can be effective in identifying Legionella as the implicated pathogen when supported by a constellation of clinical, radiographic, and laboratory parameters. Unfortunately, critical evaluation of such scoring systems has been wanting, and the few studies performed to date have yielded conflicting results $[5 \bullet, 9-11]$. Moreover, the widespread availability and fairly rapid turnaround of Legionella urinary antigen testing in recent years has steered clinicians away from the clinical syndromic approach toward protocol-driven treatment strategies tailored toward aggressive early therapeutic intervention followed by de-escalation based upon diagnostic results. The diagnostic dilemma remains, though, as urinary antigen tests for both Legionella and Streptococcus pneumoniae are imperfect in identifying many serogroups much less other pathogenic Legionella species.

\section{Microbial Ecology and Epidemiology}

Legionella species are small obligate aerobic gram-negative bacilli found in freshwater environments worldwide with fastidious growth requirements at temperatures typically between 20 and $42{ }^{\circ} \mathrm{C}$. Most closely related to Coxiella burnetii, the etiologic agent of $\mathrm{Q}$ fever, Legionella species are likewise obligate intracellular parasites. The family Legionellaceae encompasses 50 species and over 70 serogroups. Legionella pneumophila is the most common species accounting for up to $91 \%$ of human infections with serogroup 1 the dominant culprit in $84 \%$ of documented cases [12]. All Legionella species require L-cysteine and soluble iron for optimal growth, thus buffered charcoal yeast extract (BCYE) is the preferred growth medium. Unfortunately, clinical isolates demonstrate a wide range of successful recovery, varying from 15 to $90 \%$ depending on the severity of illness. After $72 \mathrm{~h}$ of incubation at $35{ }^{\circ} \mathrm{C}$ with $5 \% \mathrm{CO} 2$, grayish "ground-glass" colonies appear and are best visualized with Giemsa stain rather than Gram stain, though monoclonal immunofluorescent antibody staining is superior to all other techniques. $[3,5 \bullet, 6]$

While Legionella species survive naturally in aquatic or moist-soil environments within dozens of species of amoebae, ciliated protozoa and even slime molds, they do not naturally cause disease in humans. Infection is a result of a complex interplay of environmental and anthropogenic factors and the subsequent opportunistic infestation of human phagocytic cells such as alveolar macrophages. Key to this process is the propensity of Legionella to maintain the sessile state within biofilms particularly in a multitude of building water systems, water heaters, air conditioners, cooling towers, and even hot tubs. When water flow and pressure dynamically disturb these sessile phase bacteria, massive numbers may suddenly be released into the water. If aerosolized and aspirated by susceptible hosts then illness and pneumonia outbreaks may occur [3, 6, 8]. These biofilms also serve to promote survival and multiplication outside of host cells and have been shown to augment bacteriocidal tolerance $[7,8]$.

Given the natural aqueous habitat of Legionella species, researchers have investigated this dynamic interaction of environmental factors such as increased rainfall, humidity, flooding, and expanding industrialized populations in order to explain the apparent increase in the incidence of Legionnaires' disease. Multiple studies have now demonstrated a correlation between wet, humid weather, particularly during the summer and fall months, and sporadic cases of community-acquired Legionella pneumonia [1315]. In fact the dramatic rise of cases in the Middle Atlantic and Eastern states by $96 \%$ from 1990 to 2005 is highlighted by the meteorologic study performed by Fisman et al. in the greater Philadelphia, PA region demonstrating a significant temporal dose-response relationship between both precipitation and increased humidity with Legionella pneumonia cases $[13,16]$. Thus, improved comprehensive diagnostic testing and reporting might not solely account for the observed increased incidence reported in the United States.

Of additional concern is the increasingly identified presence of Legionella pneumophila in the potable water supply of hospitals, long-term care facilities, and nursing homes. Multiple outbreaks of nosocomial Legionella pneumonia in the United States and Europe alike have been attributed to colonization of hospital water systems, with rates as high as $70 \%$ in some geographic regions. Longterm care facilities, even newly constructed, are not immune to the risk of Legionella colonization, as the VA Health Care System discovered in an outbreak of Legionella pneumonia in 6 patients at a brand new long-term facility in Pittsburgh, PA. Microaspiration of susceptible individuals is considered the likely mechanism of transmission particularly in patients with significant neurologic impairment. Any nosocomial case should alert the clinician and compel infection control personnel to investigate building water supply contamination [17-20]. Other sources of community transmission have included contaminated water in hotels and cruise ships leading to travel-associated Legionnaires' disease as well as localized sporadic cases from spa and 
exercise facilities. Source investigation of pools and hot tubs revealed inadequate chlorination, bromination, and $\mathrm{pH}$ monitoring, in addition to the presence of Legionella biofilms among filter systems as unifying explanations in many of these outbreaks [21-24].

\section{Clinical Presentation}

In the largest recent case studies, Legionella accounts for between 4.6 and $13.9 \%$ of patients hospitalized with community-acquired pneumonia with mortality rates of treated patients approximately $5-6 \%\left[25^{\bullet}, 27\right]$. The incubation period is between 2 and 10 days though outliers with longer incubation periods up to 28 days have been reported in some outbreaks [6]. Patients have classically been described as acutely ill appearing with high fevers, lethargy, confusion, non-productive cough, relative bradycardia, and prominent gastrointestinal features. Up to $90 \%$ of individuals had fevers over $38.5{ }^{\circ} \mathrm{C}, 50 \%$ reported headaches, and between 30 and $50 \%$ presented with gastrointestinal symptoms including nausea, vomiting, and diarrhea $[2,5 \cdot, 31]$. Relative bradycardia to temperature elevation was a recurring observation leading some to promote this as a potential specific marker for Legionella pneumonia, though more rigorous evaluations failed to confirm any significant correlation $[5 \cdot, 9,11]$.

Extrapulmonary findings have also featured prominently in many early descriptions and have been espoused as a means of distinguishing Legionella from other potential pathogens including Streptococcus pneumoniae or Chlamydophila pneumoniae. Confusion, hepatic dysfunction, rhabdomyolysis, hypophosphatemia, hyponatremia, and renal impairment with hematuria and proteinuria are common manifestations reported more frequently with Legionella. Elevated lactate dehydrogenase (LDH) particularly in the absence cold agglutinin hemolytic anemia can help differentiate between Legionella and Mycoplasma pneumonia. Although non-specific, elevated creatinine phosphokinase (CPK), C-reactive protein (CRP), and Ferritin levels are all useful lab abnormalities much more exclusive to Legionnaires' disease [2, 5•, 11, 31, 32]. Chest radiography typically reveals patchy unilobar or multilobar infiltrates which progress to dense consolidations even despite appropriate antimicrobial therapy, and associated pleural effusions are not uncommon [33, 34]. Unfortunately no pathognomonic radiographic findings point to Legionella as the diagnosis as all types of infiltrates can occur including interstitial, nodular, and, in the immunocompromised, pleural-based rounded opacities, cavitary lesions, and lung abscesses [34, 35].

Cumulative data over subsequent decades have more often than not illustrated the typical protean manifestations
Table 1 Clinical, radiographic, and laboratory characteristics for Legionella CAP and non-Legionella/Pneumococcal CAP

\begin{tabular}{|c|c|c|c|}
\hline Characteristic & $\begin{array}{l}\text { Legionella } \\
\text { CAP }\end{array}$ & $\begin{array}{l}\text { Non- } \\
\text { Legionella } \\
\text { CAP }\end{array}$ & $p$ value \\
\hline \multicolumn{4}{|l|}{ Demographic } \\
\hline Age (mean) & $57-68$ & $64-73$ & $<.001-.01$ \\
\hline Male sex & $70-89 \%$ & $60-66 \%$ & $<.001-.11$ \\
\hline $\begin{array}{l}\text { Current/former } \\
\text { smoker }\end{array}$ & $51-73 \%$ & $31-57 \%$ & $<.001-.003$ \\
\hline \multicolumn{4}{|l|}{ Clinical } \\
\hline Cough & $66-70 \%$ & $88.5-90 \%$ & $<.001$ \\
\hline Purulent sputum & $27-40 \%$ & $64-72 \%$ & $<.001$ \\
\hline Myalgias/arthralgias & $43-51 \%$ & $14-16.7 \%$ & $<.001$ \\
\hline Headache & $40.7-43 \%$ & $14-15 \%$ & $<.001$ \\
\hline Pleuritic chest pain & $21-24 \%$ & $53.7-65 \%$ & $<.001$ \\
\hline Confusion & $11-25 \%$ & $8-15.7 \%$ & $.049-\mathrm{NS}$ \\
\hline $\begin{array}{l}\text { Mean temperature } \\
\left({ }^{\circ} \mathrm{C}\right)\end{array}$ & $38.5-39.3$ & $37.8-38.4$ & $<.001$ \\
\hline Heart rate (beats/min) & $100-103$ & $95-104$ & NS \\
\hline Tachycardia $>100$ & $53.5 \%$ & 56.1 & NS \\
\hline Shock & $2-2.4 \%$ & $11-12.4 \%$ & $<.001-.025$ \\
\hline GI symptoms & $19 \%$ & $10 \%$ & .007 \\
\hline \multicolumn{4}{|l|}{ Laboratory } \\
\hline Leukocyte count $>2$ & $49 \%$ & $68 \%$ & $<.001$ \\
\hline Elevated AST or ALT & $63-71 \%$ & $34-35 \%$ & $<.001$ \\
\hline $\begin{array}{l}\text { Sodium mean } \\
(\mathrm{mmol} / \mathrm{L})\end{array}$ & $132-132.5$ & $134.6-136$ & $<.001-.003$ \\
\hline LDH mean (U/L) & $252-671$ & $214-480$ & $<.001$ \\
\hline C-reactive protein & 241 & 134 & $<.001$ \\
\hline \multicolumn{4}{|l|}{ Radiographic } \\
\hline Multilobar pneumonia & $40.4-43 \%$ & $33-38 \%$ & $.03-\mathrm{NS}$ \\
\hline Pleural effusion & $14.6-23 \%$ & $20.7-26 \%$ & $.03-\mathrm{NS}$ \\
\hline
\end{tabular}

Adapted from Fernandez-Sabe et al. [10], Viasus et al. [25 ${ }^{\bullet}$ and Fiumefreddo et al. [36]

NS Not Significant

of community-acquired pneumonia as clinical syndromic diagnostic approaches or scoring systems have inconsistently borne fruit in differentiating Legionella from other bacterial causes [9-11]. However, 3 more recent studies over the past decade have illuminated many of the aforementioned trends as shown in the following summary table adapted from these publications (Table 1) [10, 25•, 36]. The comparator pathogen in all three studies was most predominantly Streptococcus pneumoniae as one would expect given it is the most common cause of communityacquired pneumonia.

In the study by Fernandez-Sabe et al., multivariate analysis identified high fevers greater than $39^{\circ} \mathrm{C}$, myalgias, and gastrointestinal symptoms as associated with Legionella pneumonia, whereas previous respiratory tract infection, pleuritic 
chest pain, and purulent sputum were associated with nonLegionella pneumonia [10]. Similarly, Fiumefreddo et al. found high fever, the absence of sputum, low Sodium, elevated LDH, and elevated CRP all predictive for Legionella pneumonia in their multivariate analysis [36].

Together these studies reinforce a number of salient points. Clinical features such as non-productive or absent cough, high fevers, myalgias, headache, and gastrointestinal symptoms and laboratory abnormalities such as low Sodium, elevated liver enzymes, LDH, and CRP favor Legionella, whereas cough with purulent sputum and pleuritic chest pain suggests Pneumococcal pneumonia as the diagnosis. However, there is significant overlap in community-acquired pneumonia presentations and, as discussed previously, scoring systems utilizing such features have yielded insufficient sensitivity and specificity to promote their use. For instance, the application of the Winthrop-University Hospital Criteria by Gupta et al., although correctly identifying Legionella with a sensitivity of $78 \%$, was less successful in exclusion with specificity of only $65 \%$ [9].

A prevailing theme of many retrospective reviews is the impact of smoking and chronic lung disease on susceptibility to Legionella pneumonia. Nearly half of patients have either chronic respiratory disease or a history of smoking, and one review demonstrated that smoking was a significant independent risk factor for ICU admission or death with an odds ratio of $2.96[25 \bullet, 26]$. Median age tends to be between 56 and 58 years of age with a male predominance in nearly every major case series, though many series also include a number of individuals under the age of 55 with no discernible risk factors [26, 27]. Other susceptible hosts include transplant recipients, particularly those receiving glucocorticoids, and patients receiving tumor necrosis factor-alpha inhibitors such as infliximab or adalimumab. Mortality rates have been over $14 \%$ in solid organ transplant patients [28-30].

Rare manifestations of Legionella have included septic arthritis, peritonitis, myocarditis, pericarditis, and nativevalve and prosthetic valve endocarditis. These unusual cases have often involved immunosuppressed patients, nosocomial infections, or non-pneumophila strains such as Legionella longbeachae or micdadei [37-44].

\section{Laboratory Diagnosis}

Because delay in appropriate diagnosis and treatment of Legionella has been shown to increase mortality and up to $20 \%$ of cases of community-acquired pneumonia are due to the atypical pathogens, prompt initiation of antimicrobial therapy including activity against these atypical pathogens is vital [45]. Diagnostic testing for the atypical pathogens and, in particular Legionella, has been problematic due to the variability and time required for recovery of these bacteria even with specialized culture techniques. Culture of Legionella on buffered charcoal yeast extract from respiratory samples is highly dependent on severity of illness with the lowest yield of 15-25\% in cases of mild pneumonia and the highest yield in cases of severe multilobar pneumonia and respiratory failure [6]. Successful culture usually takes 3-5 days but longer incubation times of up to 2 weeks may be necessary. Thus, though highly specific, culture results alone should not guide therapeutic decisions.

Legionella urinary antigen testing has improved the rapidity of diagnosis and largely supplanted serologic testing. Serologic testing with indirect fluorescent antibody (IFA) or enzyme-linked immunosorbent assays (ELISA) has sensitivity between 20 and $70 \%$ and a specificity of $95 \%$ but requires a four-fold rise $\geq 128$ in acute and convalescent titers after 8-12 weeks [6]. In contrast, the urinary antigen test is relatively simple, affordable, and widely available with turnaround times of only a few hours in many hospitals. The major disadvantage however is that commercial urinary antigen assays still only detect serogroup 1, which accounts for only 70-80\% of cases. Sensitivity has been dependent upon both the severity of illness and the quality of the study performed. A recent large metaanalysis performed by Shimada et al. revealed an overall pooled sensitivity of 0.74 with an excellent specificity of 0.99 for urinary antigen tests [46]. Direct Fluorescent antibody (DFA) staining can also be performed on respiratory specimens with high specificity. However, testing requires a high level of technical expertise and the resultant sensitivity is inconsistent between 25 and $75 \%$ [6].

PCR assays for Legionella are still in development with limited commercial availability and clinical experience is therefore lacking. Diederen et al. evaluated 37 confirmed Legionella infections with real-time PCR assays but found no improvement with $16 \mathrm{~s}$ rRNA PCR testing over urinary antigen alone. However, by utilizing the mip gene PCR, an additional 4 cases were identified [47]. More recently, Mentasti et al. investigated confirmed Legionnaires' disease cases during an outbreak with a real-time mip gene PCR assay and found 47 of $80(58.8 \%)$ culture negative respiratory samples to be positive with $100 \%$ specificity for Legionella pneumophila [48]. Further studies are underway in elucidating the clinical role of PCR testing and will need critical evaluation before routine PCR testing can be recommended. There are no FDA approved PCR tests currently available [49].

\section{Therapy}

Mortality rates in patients with untreated or inadequately treated Legionnaires' disease have been devastatingly high approaching $40 \%$ in most early outbreaks and up to $80 \%$ 
among nosocomial immunosuppressed patients [2, 32]. For most bacterial pathogens, antimicrobial susceptibility testing guides therapeutic choices, but unfortunately, in vitro susceptibility results are neither reliable nor standardized for Legionella. In addition, no large prospective randomized-controlled clinical trials have been performed for Legionnaire's disease. Only small observational prospective studies or retrospective data from community-acquired or nosocomial outbreaks are available. Because Legionella bacteria are intracellular parasites of human cells such as alveolar macrophages, antimicrobial agents must achieve sufficient intracellular concentrations and bioactivity where the bacteria reside. Beta-lactams and aminoglycosides are ineffective precisely due to their poor intracellular activity. In contrast macrolides, quinolones, tetracyclines, and rifampin are all capable of achieving intracellular concentrations higher than the minimum inhibitory concentration [MIC] against Legionella species. Investigations of early outbreaks have reinforced this paradigm as mortality rates for patients treated with tetracyclines or erythromycin were consistently lower (7-10\%) compared to patients treated with beta-lactams, aminoglycosides, or phenicols $(20-40 \%)[2,6,32]$.

The guinea pig model now serves as the primary method of testing antimicrobial efficacy for severe Legionella infection and has been shown to correlate closely with treatment of Legionnaires' disease in humans. Utilizing these models, azithromycin, newer ketolides, and fluoroquinolones demonstrate superior intracellular activity against Legionella pneumophila including more rapid bacterial clearance, reduced length of therapy, and lower dose requirements to achieve cure $[6,53]$.

A number of prospective observational studies have investigated whether macrolides or fluoroquinolones are superior in the treatment of Legionnaire's disease. Although these studies have not shown significant differences in mortality rates, the respiratory fluoroquinolones appear to result in more rapid defervescence, fewer complications, and shorter hospital stays. In one large outbreak in Spain in 2001, patients treated with levofloxacin had fewer complications (3.4 vs. $27.2 \% ; p=.02$ ) and shorter mean hospital stays (5.5 vs. 11.3 days; $p=.04$ ) compared to clarithromycin [50]. A prospective study evaluating 130 patients by Sabria et al. found faster time to defervescence (48 vs. $77.1 \mathrm{~h}$; $p<.001)$ as well as shorter mean hospital stays (7.6 vs. 9.9 days; $p=0.09$ ), and the series by Myketiuk et al. in 2005 similarly observed faster time to defervescence (2.0 vs. 4.5 days; $p<.001$ ) and clinical stability (3 vs. 5 days; $p=.002$ ) both favoring levofloxacin compared to macrolides. However, case-fatality rates and complications were not significantly different for either of these latter two studies and the macrolides used were erythromycin or clarithromycin [27, 51]. Finally, a very recent systematic meta- analysis by Burdet et al. was able to compare outcomes between quinolone and macrolide monotherapy revealing a trend toward lower mortality with quinolone use (4.0 vs. $10.9 \%$ ) and confirming shorter length of hospital stays with quinolone treatment [54•]. However, no randomized-controlled trials were available in this meta-analysis.

Combination approaches utilizing a fluoroquinolone, macrolides, or rifampin have been advocated by some authors particularly in severe pneumonia in immunosuppressed individuals or in cases of extrapulmonary Legionella such as endocarditis [32, 53]. However, in the sub-group analysis by Garrido et al., no benefit with the rifampin combination was found, and in fact an increase in adverse side effects was noted with rifampin (20 vs. $11 \%$ ) including $9 \%$ of patients who developed liver toxicity [50]. Another small study of 32 patients likewise found the combination of rifampin and clarithromycin to increase hospital stays by $50 \%$ with a trend toward increased liver toxicity [52]. Although in vitro and in vivo studies have shown synergy or partial-synergy with the combination of macrolides and quinolones, only one observational study has suggested a trend toward improved outcomes in cancer patients with Legionella pneumonia [53, 55].

For community-acquired or nosocomial Legionella pneumonia, cumulative data and expert opinion support the use of a respiratory fluoroquinolone such as levofloxacin $750 \mathrm{~m} \mathrm{mg}$ or azithromycin $1000 \mathrm{mg}$ loading dose followed by $500 \mathrm{mg}$ daily for 7-10 days. Initial intravenous therapy is reasonable until the patient defervesces and has demonstrated no gastrointestinal compromise. Patients clinically improve usually after $72 \mathrm{~h}$ though radiographic progression is not uncommon. For severely ill immunosuppressed patients, many authors recommend longer durations of treatment of up to 21 days. And in patients with endocarditis, 3-6 months of combination fluoroquinolone and macrolide therapy should be given serious consideration.

Prognosis is generally favorable in immunocompetent individuals with mortality rates consistently $<5 \%$ when appropriate antimicrobial therapy is initiated without delay. Unfortunately despite appropriate management, immunocompromised individuals such as cancer, transplant, or HIV patients continue to have unacceptably high mortality rates approaching $20 \%$ [20, 56, 57]. Quality of life among survivors can be significantly impacted as demonstrated in one long-term follow-up study with $75 \%$ of survivors reporting fatigue and $66 \%$ persistent neurologic symptoms after 17 months [58].

\section{Prevention}

Legionella species are transmitted via aerosolized or ingested contaminated water sources and cannot be 
transmitted from person to person thus isolation is not required. It is a reportable illness, and any case should be reported to the state health department for source investigative purposes. This process has been instrumental in curtailing outbreaks as demonstrated recently in the Denver, Colorado outbreak of 2 cases of pneumonia acquired from a contaminated hot tub at a local recreational fitness gym. The source was quickly identified, and inadequate bromination and contaminated hot tub filters were to blame. The equipment was quickly decontaminated, sterility confirmed, and no further cases occurred [24].

Nosocomial outbreaks occur due to contaminated water supplies and can be prevented by routine culturing of the hospital drinking water. This proactive approach advocated by Stout et al. successfully identified Legionella species in the drinking water of $70 \%$ of 20 hospitals evaluated, and in fact 4 hospitals were found to have previously unidentified nosocomial Legionella pneumonia cases [59]. This has become an increasingly attractive protocol in many bone marrow transplant centers given the high morbidity and mortality of Legionella pneumonia in these patients. However, according to the CDC, aggressive laboratory testing for Legionella in patients with nosocomial pneumonia should always be pursued, but environmental cultures should be reserved for clearly documented cases of Legionella nosocomial pneumonia [60]. Copper-silver ionization units should be employed for disinfection of hospital water supplies and protocols are described at length in manuals on the CDC government website [61].

\section{Conclusion}

Legionnaires' disease continues to be a significant threat for immunosuppressed and immunocompetent individuals alike with rising incidence rates of community-acquired pneumonia and recurrent outbreaks of both travel-associated and nosocomial pneumonia. Patients with chronic respiratory illnesses and a significant smoking history are particularly susceptible to Legionella pneumonia. Although cure rates for most patients with community-acquired pneumonia are favorable, the mortality rates for immunocompromised individuals particularly in the setting of nosocomial pneumonia remain unacceptably high. Early diagnosis utilizing a constellation of clinical findings such as high fevers, gastrointestinal symptoms and headache; laboratory abnormalities such hyponatremia, elevated $\mathrm{LDH}$, and elevated CRP; and Legionella urinary antigen testing can be successful but not foolproof in singling out Legionella as the culprit pathogen. Further research is desperately needed for more accurate and comprehensive means of identifying other pathogenic Legionella serogroups or species. Perhaps PCR-based methodologies will bear fruit in time, but until so, a high-degree of suspicion and appropriate antimicrobial therapy, preferably a respiratory fluoroquinolone directed against Legionella, should be employed. Preventative measures and close monitoring of water supplies particularly in hospital or health-care facilities will also continue to play a vital role in mitigating against future outbreaks.

\section{Compliance with Ethics Guidelines}

Conflict of Interest Dr. Robert Eison declares that he has no conflict of interest

Human and Animal Rights and Informed Consent This article does not contain any studies with human or animal subjects performed by the authors.

\section{References}

Papers of particular interest, published recently, have been highlighted as:

- Of importance

1. Tatlock H. A Rickettsia-like organism recovered from guinea pigs. Proc Soc Exp Biol Med. 1944;57:95-9.

2. Fraser DW, Tsai TR, Orenstein W, Parkin WE, Beecham HJ, Sharrar RG, Harris J, et al. Legionnaires' disease: description of an epidemic of pneumonia. N Engl J Med. 1977;297:1189-97.

3. Fields BS, Benson RF, Besser RE. Legionella and Legionnaires' disease: 25 years of investigation. Clin Microbiol Rev. 2002;215(3):506-26.

4. Hicks LA, Garrison LE, Nelson GE, Hampton LM. Legionellosis-United States, 2000-2009. MMWR. 2011;60(32):1080-6.

5. - Cunha BA. Legionnaires' disease: clinical differentiation from typical and other atypical pneumonias. Infect Dis Clin N Am. 2010;24:73-105. This comprehensive review provides arguably the most thorough description of the clinical, radiographic, and extrapulmonary manifestations of Legionella pneumonia in comparison to other sundry causes of both typical and atypical pneumonias and is a vital source of information for any clinician involved in hospital based medicine.

6. Mandell GL, Bennett JE, Dolin R. Principles and practice of infectious diseases. 6th ed. Philadelphia: Churchill Livingston; 2005. p. 2711-24.

7. Green PN, Pirrie RS. A laboratory apparatus for the generation and biocide efficacy testing of Legionella biofilms. J Appl Bacteriol. 1993;74:388-93.

8. Abdel-Nour M, Duncan C, Low DE, Guyard C. Biofilms: the stronghold of Legionella pneumophila. Int J Mol Sci. 2013;14: 21660-75.

9. Gupta SK, Imperiale TF, Sarosi GA. Evaluation of the WinthropUniversity Hospital criteria to identify Legionella pneumonia. Chest. 2001;120:1064-71.

10. Fernandez-Sabe N, Roson B, Carratala J, Dorca J, et al. Clinical diagnosis of Legionella pneumonia revisited: evaluation of the community-based pneumonia incidence study group scoring system. Clin Infect Dis. 2003;37:483-9.

11. Mulazimoglu L, Yu VL. Can Legionnaires disease be diagnosed by clinical criteria? A critical review. Chest. 2001;120:1049-53.

12. Yu VL, Plouffe JF, Pastoris MC, Stout JE, et al. Distribution of Legionella species and serogroups isolated by culture in patients 
with sporadic community-acquired legionellosis: an international collaborative survey. J Infect Dis. 2002;186(1):127-8.

13. Fisman DN, Lim S, Wellenius GA, et al. It's not the heat, it's the humidity: wet weather increases legionellosis risk in the greater Philadelphia metropolitan area. J Infect Dis. 2005;192:2066.

14. Ricketts KD, Charlett A, Gelb D, et al. Weather patterns and Legionnaires' disease: a meteorological study. Epidemiol Infect. 2009; 137:1003.

15. Garcia-Vidal C, Labori M, Viasus D, et al. Rainfall is a risk factor for sporadic cases of Legionella pneumophila pneumonia. PLoS One. 2013;8(4):e61036.

16. Neil K, Berkelman R. Increasing incidence of legionellosis in the United States, 1990-2005. Clin Infect Dis. 2008;47(5):591-9.

17. Kool JL, Bergmire-Sweat D, Butler JC, et al. Hospital characteristics associated with colonization of water systems by Legionella and risk of nosocomial legionnaires' disease: a cohort study of 15 hospitals. Infect Control Hosp Epidemiol. 1999;20(12):798-805.

18. Sabria M, Yu VL. Hospital-acquired legionellosis: solutions for a preventable infection. Lancet Infect Dis. 2002;2(6):368-73.

19. Lin YS, Yu VL, Vidic RD. Disinfection of water distribution systems for Legionella. Semin Respir Infect. 1998;13(2):147.

20. Stout JE, Brennen C, Muder RR. Legionnaires' disease in a newly constructed long-term care facility. J Am Geriatr Soc. 2000;48(12):1589-92.

21. Burnsed LJ, Hicks LA, Smithee LMK, Fields BS, et al. A large travel-associated outbreak of Legionellosis among hotel guests: utility of the urine antigen assay in confirming pontiac fever. Clin Infect Dis. 2007;44(2):222-8.

22. Benin AL, Bwenson RF, Arnold KE, Fiore AE, et al. An outbreak of travel-associated legionnaires disease and pontiac fever: the need of enhanced surveillance of travel-associated Legionellosis in the United States. J Infect Dis. 2002;185(2):237-43.

23. Joseph CA, Yadav R, Ricketts KD. Travel-associated Legionnaires disease in Europe 2007. Euro Surveill. 2009;14(18):19196.

24. Personal Communication. Thomas Butts, Deputy Director of Tricounty Health Department. Denver, CO, May 2014.

25. • D Viasus, S Di Yacova, C Garcia-Vidal et al. Community-acquired Legionella pneumophila pneumonia: a single-center experience with 214 hospitalized sporadic cases over 15 years. Medicine (Baltimore). 2013;92(1):51-60. This excellent study is one of the largest retrospective series detailing the clinical characteristics and outcomes for patients hospitalized with Legionella pneumonia.

26. Yu VL, Greenberg RN, Zadeikis N, et al. Levofloxacin efficacy in the treatment of community-acquired legionellosis. Chest. 2004; 125(6): 2135.

27. Mykietiuk A, Carratala J, Fernandez-Sabe N, et al. Clinical outcomes for hospitalized patients with Legionella pneumonia in the antigenuria era: the influence of levofloxacin therapy. Clin Infect Dis. 2005;40:794-9.

28. Singh N, Stout JE, Yu VL. Prevention of Legionnaires' disease in transplant recipients: recommendations for a standardized approach. Transpl Infect Dis. 2005;6(2):58-62.

29. Lanternier F, Tubach F, Ravaud P, Salmon D, et al. Incidence and risk factors of Legionella pneumophila pneumonia during antitumor necrosis factor therapy: a prospective French study. Chest. 2013; 144(3):990-8.

30. Gudiol C, Garcia-Vidal C, Fernandez-Sabe N, et al. Clinical features and outcomes of Legionnaires disease in solid organ transplant recipients. Transpl Infect Dis. 2009;11(1):78-82.

31. Jespersen S, Sogaard OS, Schonheyder HC, et al. Clinical features and predictors of mortality in admitted patients with community-acquired and hospital-acquired legionellosis: a Danish historical cohort study. BMC Infect Dis. 2010;10:124.

32. Kirby BD, Snyder KM, Meyer RD, Finegold SM. Legionnaires' disease: report of sixty-five nosocomially acquired cases and review of the literature. Medicine (Baltimore). 1980;59(3):188.
33. Tan MJ, Tan JS, Humor RH, et al. The radiographic manifestations of Legionnaires' disease from the initial presentation on admission to recovery using strict criteria for the diagnosis of infection. Chest. 2000;117(2):398-403.

34. Muder RR, Yu VL, Parry MF. The radiologic manifestations of Legionella pneumonia. Semin Respir Infect. 1987;2(4):242-54.

35. Hui Y, Futoshi H, Koide M, et al. Lung abscess caused by Legionella species: implication of the immune status of hosts. Intern Med. 2009;48(23):1997-2002.

36. Fiumefreddo R, Zaborsky R, Haeuptle J, et al. Clinical predictors for Legionella in patients presenting with community-acquired pneumonia to the emergency department. BMC Pulm Med. 2009; 9(4):2466.

37. Andereya S, Schneider U, Siebert CH, Wirtz DC. Reactive knee and ankle joint arthritis: abnormal manifestation of Legionella pneumophila. Rheumatol Int. 2004;24(3):182-4.

38. Bemer P, Leautaz S, Ninin E, et al. Legionella pneumophila arthritis: use of medium specific for Mycobacteria for isolation of L. pneumophila in culture of articular fluid specimens. Clin Infect Dis. 2002;35(1):E6.

39. Lowry PW, Tompkins LS. Nosocomial legionellosis: a review of pulmonary and extrapulmonary syndromes. Am J Infect Control. 1993;21(1):21.

40. Larive S, Nguyen HT, Auvray E, et al. Acute pericarditis of unusual etiology. Rev Pneumol Clin. 1998;54(3):152-5.

41. Muder RR, Yu VL. Infection due to Legionella species other than L. pneumophila. Clin Infect Dis. 2002;35(8):990-8.

42. Leggieri N, Gouriet F, Thuny F, et al. Legionella longbeachae and endocarditis. Emerg Infect Dis. 2012;18(1):95-7.

43. Fukuta Y, Yidiz-Aktas IZ, Pasculle AW, Veldkamp PJ. Legionella micdadei prosthetic valve endocarditis complicated by brain abscess: case report and review of the literature. Scand J Infect Dis. 2012;44(6):414-8.

44. Samuel V, Bajwaa AA, Curry JD. First case of Legionella pneumophila native valve endocarditis. Int J Infect Dis. 2011; 15(11): $576-7$.

45. Heath CH, Grove DI, Looke DF. Delay in appropriate therapy of Legionella pneumonia associated with increased mortality. Eur J Clin Microbiol Infect Dis. 1996;15(4):286.

46. Shimada T, Noguchi Y, Jackson JL, et al. Systematic review and metaanalysis: urinary antigen tests for Legionellosis. Chest. 2009; 136(6):1576-85.

47. Diederen B, Kluytmans J, Vandenbroucke-Grauls C, Peeters MF. Utility of real-time PCR for diagnosis of Legionnaires' disease in routine clinical practice. J Clin Microbiol. 2008; 46(2):671-7.

48. Mentasti M, Fry NK, Afshar B, et al. Application of Legionella pneumophila-specific quantitative real-time PCR combined with direct amplification and sequence-based typing in the diagnosis and epidemiological investigation of Legionnaire's disease. Eur J Clin Microbiol Infect Dis. 2012;31(8):2017-28.

49. Maze MJ, Slow S, Cumins AM, et al. Enhanced detection of Legionnaires' disease by PCR testing of induced sputum and throat swabs. Eur Respir J. 2014;43(2):644-6.

50. Blasquez Garrido RM, Espinosa Parra FJ, Alemany Frances L, et al. Antimicrobial chemotherapy for Legionnaires' disease: levofloxacin versus macrolides. Clin Infect Dis. 2005;40(6):800-6.

51. Sabria M, Pedro-Botet ML, Gomez J, et al. Fluoroquinolones versus Macrolides in the treatment of Legionnaires' disease. Chest. 2005;128(3):1401-5.

52. Varner TR, Bookstaver PB, Ruydisill CN, Albrecht H. Role of rifampin-based combination therapy for severe communityacquired Legionella pneumophila pneumonia. Ann Pharmacother. 2011;45(7-8):967-76.

53. Pedro-Botet ML, Yu VL. Treatment strategies for Legionella infection. Expert Opin Pharmacother. 2009;10(7):1109-21. 
54. - Burdet C, Lepeule R, Duval X, et al. Quinolones versus macrolides in the treatment of legionellosis: a systematic review and meta-analysis. J Antimicrob Chemother. 2014;69(9):2354-60. This very recent extensive meta-analysis provides an excellent summary of the many studies comparing therapeutic outcomes for the treatment of Legionella pneumonia.

55. Jacobson KL, Miceli MH, Tarrand JJ, Kontoyiannis DP. Legionella pneumonia in cancer patients. Medicine (Baltimore). 2008;87(3):152-9.

56. Pedro-Botet ML, Sabria-Leal M, Sopena N, et al. Role of immunosuppression in the evolution of Legionnaires' disease. Clin Infect Dis. 1998;26(1):14-9.
57. Pedro-Botet ML, Sabria-Leal M, Sopena N, et al. Legionnaires' disease and HIV infection. Chest. 2003;124:543-7.

58. Lettinga KD, Verbon A, Nieuwkirk PT, et al. Health-related quality of life and posttraumatic stress disorder among survivors of an outbreak of Legionnaires' disease. Clin Infect Dis. 2002;35(1):11-7.

59. Stout JE, Muder RR, Mietzner S, et al. Role of environmental surveillance in determining the risk of hospital-acquired legionellosis: a national surveillance study with clinical correlations. Infect Contro Hosp Epidemiol. 2007;28(7):818-24.

60. Pedro-Botet ML, Pedro-Botet VL ML, YuVL. Treatment and prevention of Legionella infection. UpToDate ${ }^{\circledR}$. June 7, 2013.

61. http://www.cdc.gov/legionella/health-depts/inv-tools.html. 